\title{
The role of apoptosis in Caenorhabditis elegans neuronal differentiation
}

\author{
WU KaiDa ${ }^{1,2}$, TIAN Dong ${ }^{1, *}$, ZHU ZhiWen $^{1}$, CHAI YongPing ${ }^{1} \&$ OU GuangShuo ${ }^{1}$ \\ ${ }^{1}$ Tsinghua-Peking Center for Life Sciences, School of Life Sciences, Tsinghua University, Beijing 100084, China \\ ${ }^{2}$ The High School Affiliated to Renmin University, Beijing 100080, China
}

Received August 20, 2015; accepted September 29, 2015; published online October 23, 2015

Citation: Wu KD, Dong T, Zhu ZW, Chai YP, Ou GS. The role of apoptosis in Caenorhabditis elegans neuronal differentiation. Sci China Life Sci, 2015, 58: 1163-1166, doi: 10.1007/s11427-015-4952-y

Dear Editor,

Neuronal apoptosis is considered to be essential for brain development and neurodegenerative disorders and has been a major focus in cell biological and neuroscientific studies since its first recognition a century ago [1]. Remarkable progress has been made in defining the molecular and cellular pathways underlying neuronal apoptosis during the past decade [2], however, it remains relatively unclear about the physiological functions of apoptosis in neural development. The neurotrophin hypothesis proposes that immature neurons compete for trophic factors that are derived from target neurons in limited supply and that only neurons that successfully establish synaptic connections with their targets would receive trophic support to survive [3]. Although this model is appealing in predicting neuronal survival, it does not explain how to trigger neuronal apoptosis in the absence of trophic factors. Moreover, the neurotrophin hypothesis may not apply to the model organism such as Caenorhabditis elegans in which the canonic nerve growth factors have not been reported yet.

Alternative models can be valuable to illustrate the physiological function of neuronal apoptosis. Among the 1,090 somatic cells generated during the development of the C. elegans hermaphrodite, 131 cells undergo programmed cell death [4]; and 105 of the 131 apoptotic cells are derived from neuronal cell lineages. Intriguingly, many neuronal

*Corresponding author (email: tian-d14@mails.tsinghua.edu.cn) apoptotic events in C. elegans are coupled with asymmetric cell divisions (ACDs): neuroblast asymmetric division produces a small daughter cell that undergoes apoptosis and a large daughter cell that differentiates or divides and differentiates into neurons (Figure 1A for Q neuroblast lineages). The recent finding about the segregation of aggresomes during asymmetric divisions suggests another possible model for the function of neuronal apoptosis [5]. Perhaps in C. elegans neuroblast lineages, misfolded proteins asymmetrically segregate to the daughter cell that is destined to die. Similarly, factors that may inhibit neuronal differentiation may be asymmetrically inherited by the apoptotic daughter cell for degradation. The "asymmetric segregation" model would assume that neuronal apoptosis removes misfolded protein or differentiation inhibitors, maintaining the fitness of neurons or promoting neuronal differentiation. This model can be examined by inhibiting neuroblast cytokinesis: if aggresomes or differentiation inhibitors could not be segregated, the defects of neuronal differentiation or function should be expected in adult nematodes. However, this model was previously difficult to be tested because of the lack of genetic tools to manipulate neuroblast cytokinesis in live animals.

To provide some tangible evidence for the "asymmetric segregation" hypothesis, we here applied two approaches to generate conditional mutations or a weak mutant allele that disrupts neuroblast cytokinesis but allows animal to survive (Figure 1B). We chose to target an evolutionarily conserved cytokinetic scaffold protein Anillin/ani-1 in C. elgeans $\mathrm{Q}$ 


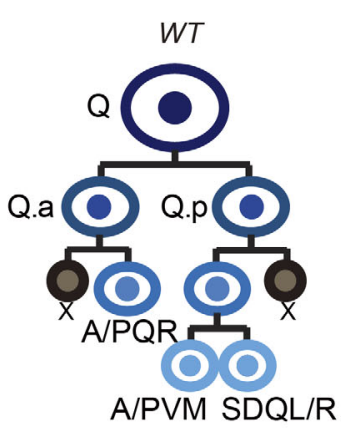

C

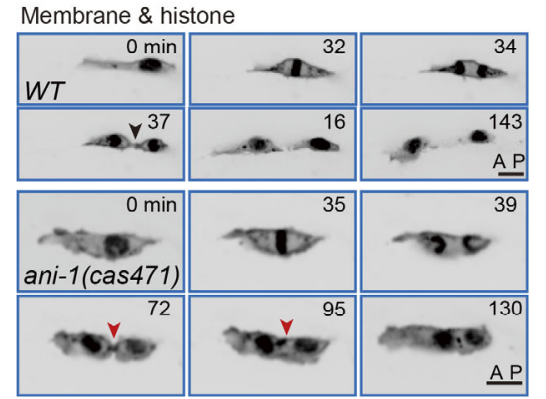

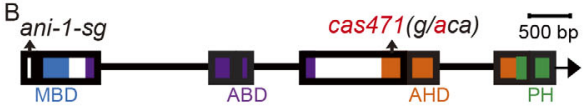
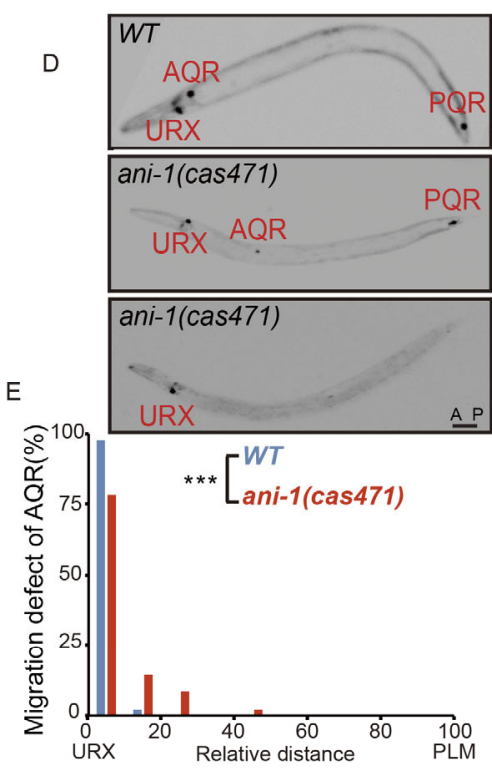

F
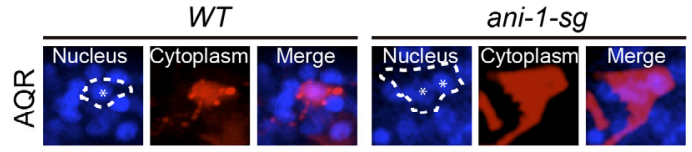

ani-1(cas471)
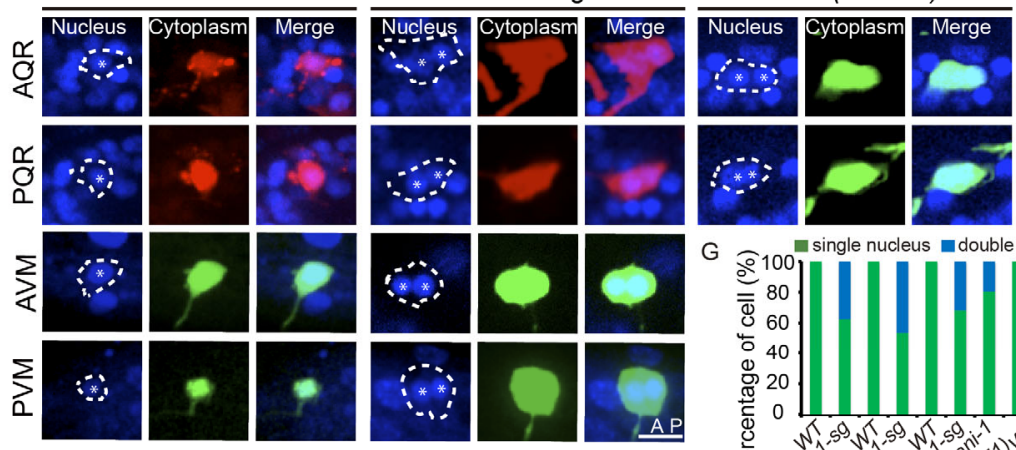

$\mathrm{G}$ single nucleus adouble nucleus
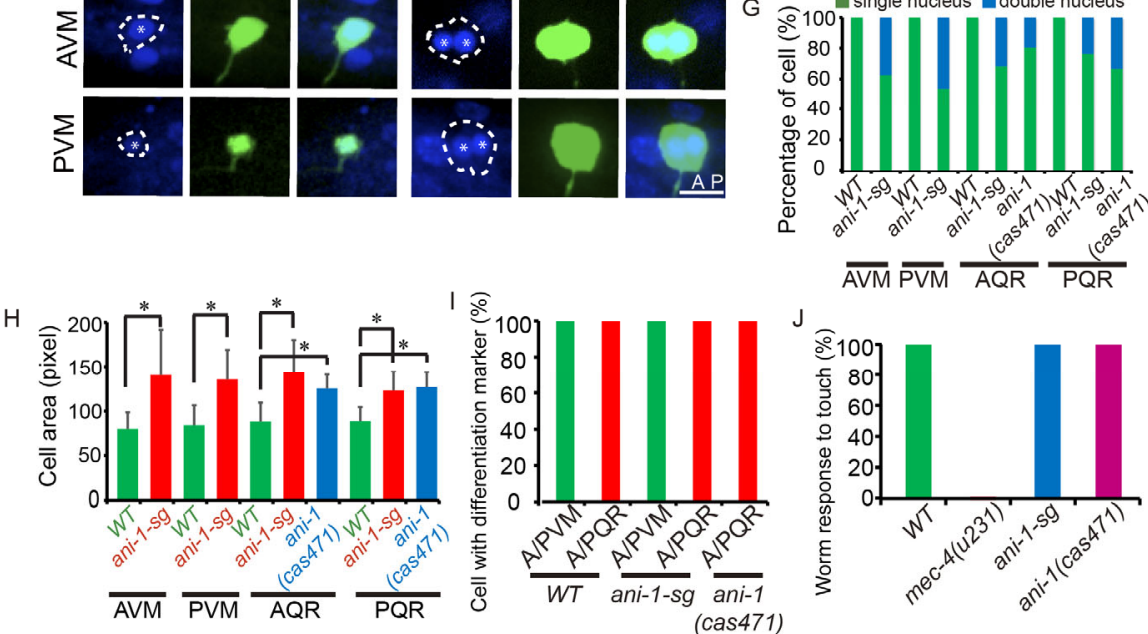

Figure 1 A, Schematic of $C$. elegans $\mathrm{Q}$ neurobloast lineages. After three rounds of asymmetric cell divisions, QL neuroblast at the left side of $C$. elegans produces PQR, PVM, SDQL and two apoptotic cells, whereas QR generates AQR, AVM, SDQR and two apoptotic cells. B, ani-1 gene model. Arrows indicate sgRNA target site (ani-1-sg) and a mutant allele (cas471). MBD, myosin-binding domain; ABD, actin-binding domain; AHD, Anillin homology domain. $\mathrm{C}$, Still fluorescence inverted images show QR cell division in WT or ani-1 mutant. Black or red arrowheads indicate the contractile ring in WT or ani-1 mutant, respectively. Plasma membrane and histone are labeled by mCherry. D, Representative images of the A/PQR position in WT and ani-1 (cas471). The middle panel shows reduced neuronal migration and the lower shows the absence of mCherry fluorescence in A/PQR neurons, which are labeled using Pgcy-32::mCherry. E, Quantification of AQR migration defect in ani-1 (cas471) mutants. F, Fluorescence images of Q neurons. Plasma membrane is labeled by GFP or mCherry and nuclei are stained by Hoechst 33342. Dotted lines manifest the outline of the cells and asterisks show the nuclei. G, Quantification of Q neurons with double nucleus in ani-1-sg and ani-1 (cas471). H, Quantification of Q neurons cell area in WT, ani-1-sg and ani-1 (cas471). *, P<0.05 based on Student's $t$-test. I, Quantification of Q neurons with differentiation marker. A/PVM are labeled by Pmec-4::gfp and A/PQR are marked using Pgcy-32::mCherry or Pgcy-32::gfp. J, Quantification of touch responses. Heads of young adult C. elgans were touched by picker to examine their reverse movements. Scale bar, $5 \mu \mathrm{m}$ in $\mathrm{C}$ and $\mathrm{F}$, or $50 \mu \mathrm{m}$ in D. 
neuroblast lineages. Anillin was demonstrated to interact with various furrow components such as actomyosin, microtubules and the plasma membrane and regulate furrow positioning and midbody formation during cytokinesis [6]. Our recent study reported that conditional mutations of Anillin in neuronal lineages can be created by expressing the CRISPR-Cas9 system with lineage-specific or inducible promoters [7]. However, one caveat of using somatic CRISPR-Cas9 technique was that other genomic loci might be edited because of the potential off-target cleavages of the Cas9 endonuclease, making it possible that the phenotypes in ani-1-sg mutant animals was indirectly caused by the loss of Anillin.

To better define the specific function of Anillin in neural development, we obtained a genetic allele of ani-1 (cas471) from our forward genetic screen for mutants defective in $\mathrm{Q}$ cell migration. Using single-nucleotide polymorphism and whole-genome sequencing, we showed that a G2365A nucleotide substitution occurs in ani-1 (cas471), which changes a highly conserved Ala789 to Thr in the RhoA binding domain (RBD) of Anillin. A recent structural biological study revealed that human Anillin Ala740 (corresponding to $C$. elegans Anillin Ala789) at the interaction surface of RBD was critical for the binding between Anillin and RhoA and that A740D mutation disrupted their interaction in vitro [8]. It is thus plausible that A789T mutation in ani-1 (cas471) mutant animals weakens the binding of Anillin to RhoA in C. elegans. Consistent with this notion, our live imaging analysis of $\mathrm{Q}$ cell cytokinesis demonstrated that the final step of cytokinesis was defective in ani-1 (cas471) mutants: the furrow contraction began and constricted as normal as Q cells in WT animals, but the cleavage of furrow regressed (Figure 1C, $130 \mathrm{~min}$; Supporting Information Movie S1, S2). In addition to cytokinesis failure, we showed that $23 \%$ of AQR neurons $(N=51)$ reduced their anterior migration (Figure 1E). Both abnormalities in cytokinesis and cell migration were previously observed in ani-1-sg conditional knockout animals. Thus, we applied two independent approaches to generate two distinct types of mutations of Anillin that inhibit cytokinesis during Q cell asymmetric division.

Next we examined the neuronal differentiation in adult animals of which Q cell failed to complete cytokinesis. To visualize two oxygen sensory neurons $A Q R$ and $P Q R$ which are derived from Q.a asymmetric cell divisions, we used Pgcy-32::mCherry or Pgcy-32::gfp as a neuronal fatespecific reporter. Similarly, we followed the fate of two mechanosensory neurons AVM and PVM, which are progenies of Q.p lineages using a Pmec-4::gfp reporter. To track nuclei in these animals, we chemically fixed them with poly formaldehyde (PFA) and stained the DNA with Hoechst 33342. In ani-1-sg mutants generated by somatic CRISPR-Cas9 method, we found that 38\% AVM, $46 \%$ PVM, 32\% AQR and 24\% PQR neurons contained two nuclei (Figure 1G), suggesting their failures of cytokinesis.
Intriguingly, all these neurons with two nuclei properly expressed the Pgcy-32::mCherry reporter in $\mathrm{A} / \mathrm{PQR}$ or Pmec-4::gfp in A/VM respectively, identical to these neurons containing only one nucleus. We noticed that the single and double-nucleated neurons have different cell sizes: the double-nucleated neurons appeared to be 1.4-1.8 times larger than the single nucleated neuron, consistent with the view that polyploidy cells are usually larger than the diploid cells. Using ani-1 (cas471) allele, we further confirmed that the correct neuronal-fate markers are expressed in the AQR and PQR neurons that contain two nuclei and that these polyploidy neurons are also about 1.4 times larger than single nucleated neurons (Figure $1 \mathrm{H}$ ).

In addition to the proper differentiation of the neural progenitors which fail to complete cytokinesis, we found that $15 \%$ ani- 1 -sg conditional mutants $(N=50)$ or $10 \%$ ani- 1 (cas471) animals $(N=50)$ did not express the correct neuronal markers in either $\mathrm{A} / \mathrm{PQR}$ or $\mathrm{A} / \mathrm{VM}$ neurons (Figure $1 \mathrm{D}$, lower panel). There are two possibilities responsible for the absence of the markers in adult animals defective in $\mathrm{Q}$ cell cytokinesis: $\mathrm{Q}$ cells which failed in cytokinesis undergo apoptosis or these bi-nucleated Q cells did not express the neuronal specific reporters, indicating the failure of neuronal differentiation. We thus performed live imaging analysis to follow the cell fate after the failure of cytokinesis in Q.a cells. Normally, the Q cell descendants that are destined to die round up within $1 \mathrm{~h}$ and are then engulfed and degraded by the neighboring hyp7 cell in another hour. However, we did not notice any obvious roundup behavior for these bi-nucleated Q cells ( $N=18$ for ani-1-sg; $N=15$ for ani-1 (cas471)). instead our time-lapse recording showed that these cells migrate and form neurite-like structure as those mono-nucleated WT cells. Our data thus favors the possibility that bi-nucleated $\mathrm{Q}$ cell progenies are defective in differentiation, resulting in the absence of functional neurons in adult animals. However, we could not exclude the probability that bi-nucleated Q cells eventually die after days as our time-lapse microscopy can only follow these cells in developing larvae for 3-4 h. Nevertheless, we showed that the bi-nucleated neurons have the potential to survive (Figure 1G). Together, our results indicated that the asymmetric cell division and neuronal apoptosis play roles in neuronal differentiation, suggesting that the asymmetric segregation model can be used to explain the function of neuronal apoptosis in C. elegans neural differentiation.

In this study, we used two fluorescence reporters to examine neuronal differentiation. Due to the technical limitations, we could not isolate individual neurons and perform the single-cell mRNA sequencing to have a comprehensive examination of the alteration in neuronal fates in ani-1 mutants. However, mec-4 encodes a master transcription factor that regulates the fate of the mechanosensory neurons, making it a faithful readout for differentiation. The examination of fluorescence reporters may not be sufficient to determine whether a neuron properly differentiates and the 
future study can apply electrophysiological analysis to assay the activity of these neurons. We have investigated the consequence of polyploidy in neuronal function using touch-based behavior assay: all the WT animals respond to and move away from the touch, whereas all the mec-4 mutant animals fail to respond as reported previously (Figure 1J). In ani-1-sg or ani-1 (cas471) animals, we did not detect any MEC phenotype, regardless of the expression of Pmec-4::gfp reporter (Figure 1J), which is consistent with the notion that other mechanosensory neurons than A/PVM may compensate for their losses. Neural development involves neuroblast asymmetric division, migration, apoptosis and differentiation. It appears that neuronal differentiation is more sensitive to the failures of asymmetric cell division and apoptosis than the defect of neuronal migration. For example, a single transmembrane protein MIG-13 specifically regulates the anterior migration of $\mathrm{QR}$ neuroblast descendants [9]; and the AQR and AVM fail to reach their correct positions in mig-13 mutants but they appear to properly differentiate by examining the Pmec-4::gfp reporter $(N>1,000)$.

In summary, this study attempted to study the roles of neuronal apoptosis during neural development in C. elegans. By using ani- 1 conditional mutations and a weak allele, we showed that the failures of $\mathrm{Q}$ neuroblast asymmetric division and apoptosis cause defects during neuronal differentiation. Although these observations can be explained by different hypotheses, the asymmetric segregation and subsequent degradation of factors that inhibit neuronal differentiation could be a plausible mechanism. We speculate that the autonomous inhibitors may be denatured proteins, which affect the fitness of the neuronal progenitors or transcriptional factors that suppress neuronal differentiation. Multiple hypotheses have been proposed to explain functional relevance of apoptosis during development [5]. First of all, the removal of excessive cells should be less risky than not generating sufficient healthy cells. Second, apoptotic cells may have the potential to acquire new features if they are allowed to survive, reminiscent of the event that a pseudogene gains a novel function by mutations. Indeed, an apoptotic cell often acquires a similar fate as its viable sister cell in ced-3 mutants which are defective in Caspase-3 [10]. Finally, our study suggests that autonomous inhibitors may be asymmetrically segregated into apoptotic cells for degradation, highlighting a potential role of apoptosis in neural development.

1 Yuan J, Yankner BA. Apoptosis in the nervous system. Nature, 2000, 407: 802-809

2 Horvitz HR. Genetic control of programmed cell death in the nematode Caenorhabditis elegans. Cancer Res, 1999, 59: 1701s-1706s

3 Bibel M, Barde YA. Neurotrophins: key regulators of cell fate and cell shape in the vertebrate nervous system. Gene Dev, 2000, 14: 2919-2937

4 Sulston JE, Horvitz HR. Post-embryonic cell lineages of the nematode, Caenorhabditis elegans. Dev Biol, 1977, 56: 110-156

5 Singhvi A, Garriga G. Asymmetric divisions, aggresomes and apoptosis. Trends Cell Biol, 2009, 19: 1-7

6 Glotzer M. The molecular requirements for cytokinesis. Science, 2005, 307: 1735-1739

7 Tian D, Diao M, Jiang Y, Sun L, Zhang Y, Chen Z, Huang S, Ou G. Anillin Regulates Neuronal Migration and Neurite Growth by Linking RhoG to the Actin Cytoskeleton. Curr Biol, 2015, 25: 1135-1145

8 Sun L, Guan R, Lee IJ, Liu Y, Chen M, Wang J, Wu JQ, Chen Z. Mechanistic insights into the anchorage of the contractile ring by anillin and Mid1. Dev Cell, 2015, 33: 413-426

9 Wang X, Zhou F, Lv S, Yi P, Zhu Z, Yang Y, Feng G, Li W, Ou G. Transmembrane protein MIG-13 links the Wnt signaling and Hox genes to the cell polarity in neuronal migration. Proc Natl Acad Sci USA, 2013, 110: 11175-11180

10 Avery L, Horvitz HR. A cell that dies during wild-type C. elegans development can function as a neuron in a ced-3 mutant. Cell, 1987, 51: $1071-1078$

Open Access This article is distributed under the terms of the Creative Commons Attribution License which permits any use, distribution, and reproduction in any medium, provided the original author(s) and source are credited.

\section{Supporting Information}

The cytokinesis of QR neuroblast in WT (Movie S1) and ani-1(cas471) mutant animal (Movie S2). Fluorescence time-lapse movies of mCherry-tagged plasma membrane and histone. Frames were taken every 60 seconds. The display rate is 7 frames per second.

Supplemental materials and methods

The supporting information is available online at life.scichina.com and link.springer.com. The supporting materials are published as submitted, without typesetting or editing. The responsibility for scientific accuracy and content remains entirely with the authors. 